\title{
Pine cone scale-inspired motile origami
}

\author{
Kahye Song ${ }^{1}$ and Sang Joon Lee ${ }^{1,2}$
}

Stimuli-sensitive hydrogels have received attention because of their potential applications in various fields. Stimuli-directed motion offers many practical applications, such as in drug delivery systems and actuators. Directed motion of asymmetric hydrogels has long been designed; however, few studies have investigated the motion control of symmetric hydrogels. We designed a pine cone scale-inspired movable temperature-sensitive symmetric hydrogel that contains $\mathrm{Fe}_{3} \mathrm{O}_{4}$. Alignment of $\mathrm{Fe}_{3} \mathrm{O}_{4}$ along the magnetic force is key in motion control in which $\mathrm{Fe}_{3} \mathrm{O}_{4}$ acts like fibers in a pine cone scale. Although a homogeneous temperature-sensitive hydrogel cannot respond to a temperature gradient, the $\mathrm{Fe}_{3} \mathrm{O}_{4}$-containing hydrogel demonstrates considerable bending motion. Varying degrees and directions of motion are easily facilitated by controlling the amount and alignment angle of the $\mathrm{Fe}_{3} \mathrm{O}_{4}$. The shape of the hydrogel layer also influences the morphological structure. This study introduced facile and low-cost methods to control various bending motions. These results can be applied to many fields of engineering, including industrial engineering. NPG Asia Materials (2017) 9, e389; doi:10.1038/am.2017.79; published online 16 June 2017

\section{INTRODUCTION}

Stimuli-responsive hydrogels have received considerable attention in recent decades because of their potential application in the development of multifunctional devices. Various types of stimuli-responsive hydrogels with applications in engineering have been introduced ${ }^{1-7}$. Stimuliresponsive hydrogels can change their shape, surface characteristics, solubility and form. These hydrogels have been used in various practical applications such as in drug delivery systems, cell culture, sensors, pumps and actuators ${ }^{7-11}$. They have been elaborately developed using various fabrication methods to broaden their applications ${ }^{12,13}$.

One of the most important advancements in this field is the application of stimuli-responsive hydrogels in motion control in smart functional materials. Stimuli-responsive hydrogels have typically been utilized for motion control, especially in actuators ${ }^{14-20}$. Motion control is also important in many microscale engineering applications such as flow control in micro-fluidic devices and design of micro actuators $^{14,21,22}$. The shape transformation of soft materials also has potential applications in tissue engineering ${ }^{23}$ : differential swelling or shrinkage creates internal stresses in the composite hydrogel sheet and transforms its shape in a specific manner. Biocompatible bilayer structures are used to fabricate tunable micro-capsules applied as robust micro-carriers ${ }^{24}$. Controlled motion of functional materials has various technical applications. Most developed movable hydrogels have anisotropic structures. A typical method of inducing motion is the combination of two different materials into a bilayer structure ${ }^{25-29}$. Owing to the varying volume changes of the two materials, the bilayer exhibits bending/unbending motion.

Designing a symmetric structure that can easily control various motions at a low cost will enable diverse new applications.
The bending mechanism in pine cone scales can provide a potential solution to this problem (Figure 1a). Pine cones open their scales in sunny dry weather and close them in humid conditions ${ }^{30}$. Pine cone scales consist of dead cells, and thus, their motion is caused by morphological changes. Different orientations of sclereids and fibers in pine cone scales affect the movement of the scale (Figure $1 b)^{30}$. The angle between the long axis of the cells and the winding direction of cellulose in the sclereids is higher than that between the long axis of cells and the winding direction of cellulose in the fibers. When scales absorb water, their tissues expand anisotropically in a direction perpendicular to the direction of the fibrils $s^{31,32}$. The asymmetric orientation of cellulose fibrils at the organ level converts local swelling into a global bending movement ${ }^{33,34}$. Macroscopic structural changes are caused by these humidity-induced microscopic strains exerted on the cells.

In addition, pine cone scales fully protect the pine seeds on rainy days. On rainy days, stiff and flat pine cone scales are folded and turned into a curved shape. Given that scales fit perfectly together like a jigsaw puzzle, there is no gap between them. The petals of a flower bud also have a curved shape, but the curvature decreases after blooming. How does this occur?

In this study, the anisotropic motion of poly $(N$-isopropylacrylamide) (PNIPAAm) layers containing $\sim 100 \mathrm{~nm} \mathrm{Fe}{ }_{3} \mathrm{O}_{4}$ powder inspired by the fibers of pine cone scales is fabricated. At the beginning, the temperature-sensitive PNIPAAm hydrogel containing $\mathrm{Fe}_{3} \mathrm{O}_{4}$ powder was randomly scattered (Figure 1c). However, when a magnetic force was applied to the layers, the $\mathrm{Fe}_{3} \mathrm{O}_{4}$ powder was aligned along the direction of magnetic force (Figure 1d). The $\mathrm{Fe}_{3} \mathrm{O}_{4}$ powder was then aggregated with PNIPAAm and stiffened (Supplementary Figures $\mathrm{S} 1 \mathrm{a}$ and $\mathrm{b}$ ). The aligned $\mathrm{Fe}_{3} \mathrm{O}_{4}$ powder acts like pine cone

\footnotetext{
${ }^{1}$ School of Interdisciplinary Bioscience and Bioengineering, Pohang University of Science and Technology (POSTECH), Gyeongbuk, Korea and ${ }^{2}$ Department of Mechanical Engineering, Pohang University of Science and Technology (POSTECH), Gyeongbuk, Korea

Correspondence: Professor SJ Lee, Department of Mechanical Engineering, Pohang University of Science and Technology (POSTECH), Room 210, Mechanical Engineering Experimental Building, 77 Cheongam-Ro, Nam-Gu, Pohang 37673, Gyeongbuk, Korea.

E-mail: sjlee@postech.ac.kr

Received 16 February 2017; revised 27 March 2017; accepted 28 March 2017
} 

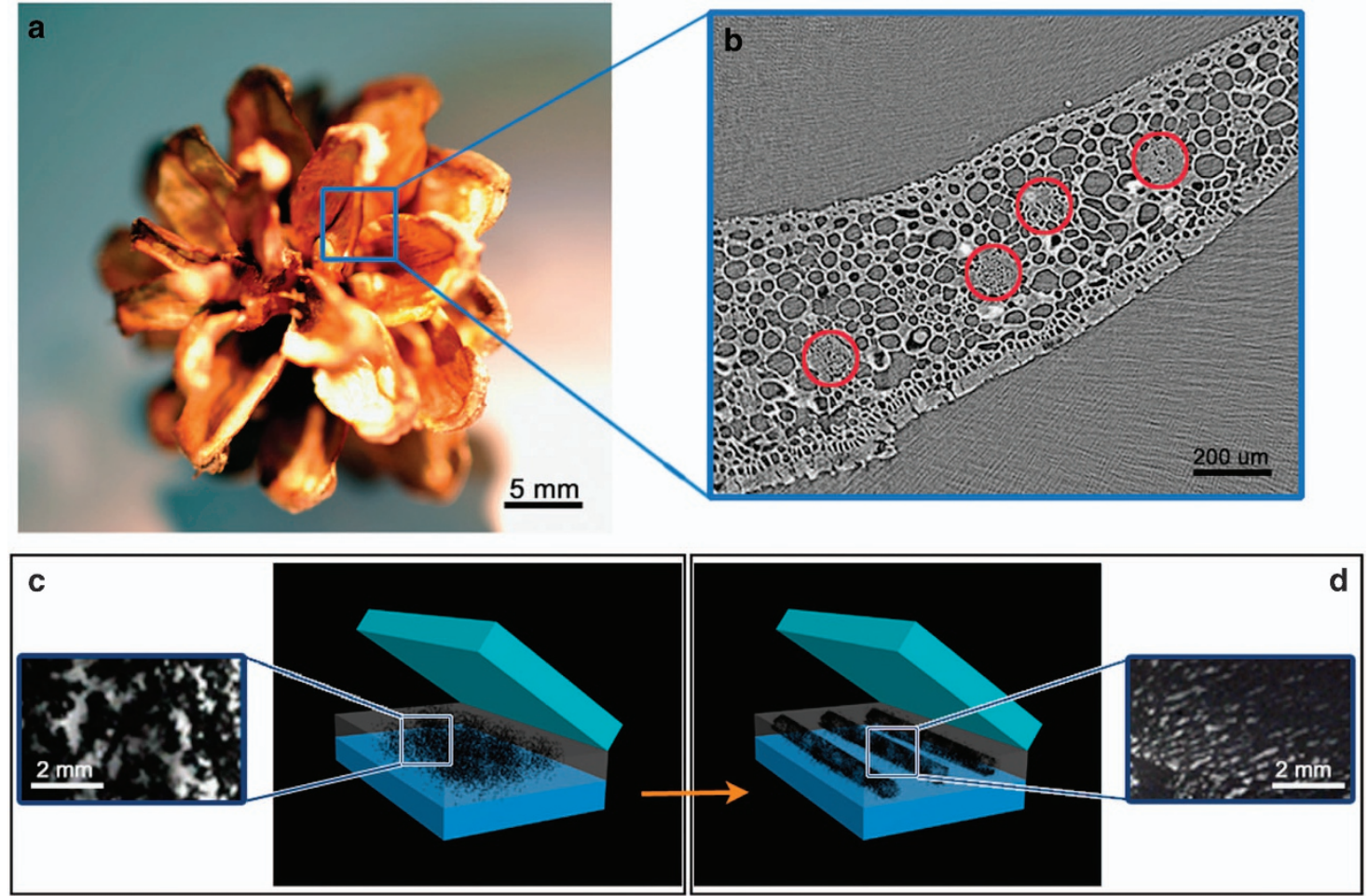

Figure 1 Asymmetric movable hydrogel layer inspired by pine cone scales. (a) Photograph of a seed-bearing pine cone. Pine seeds are housed in the scales. The blue square indicates the pine cone scales. (b) Cross-sectional image of a pine cone scale acquired using X-ray microscopy. The red circle indicates the fibers. (c) $\mathrm{Fe}_{3} \mathrm{O}_{4}$ powder dispersed on a PNIPAAm layer. The figures were created using SolidWorks software (Dassault Systèmes SolidWorks Corp., Waltham, MA, USA). (d) The $\mathrm{Fe}_{3} \mathrm{O}_{4}$ powder is aligned with the PNIPAAm layer using a magnetic field. The system schematics were created using SolidWorks software. PNIPAAm, poly( $N$-isopropylacrylamide).

fibers and facilitates the asymmetric motion of the PNIPAAm layers: the triple layer can bend repetitively in the fixed direction.

Even after polymerization, because the PNIPAAm does not have magnetic properties, the magnetism of the PNIPAAm and $\mathrm{Fe}_{3} \mathrm{O}_{4}$ mixture is similar to that of pure $\mathrm{Fe}_{3} \mathrm{O}_{4}$ powder: the top moment of $1 \mathrm{~g}$ of $\mathrm{Fe}_{3} \mathrm{O}_{4}$ powder is $\sim 76.8$ e.m.u. $\mathrm{g}^{-1}$, and that of PNIPAAm with $1 \mathrm{~g}$ of $\mathrm{Fe}_{3} \mathrm{O}_{4}$ powder is $\sim 73.6$ e.m.u. $\mathrm{g}^{-1}$ (Supplementary Figure S1c). These slight differences occurred due to the nonuniform powder distribution. The PNIPAAm does not have magnetic properties that affect the magnetic hysteresis of $\mathrm{Fe}_{3} \mathrm{O}_{4}$ powder. Thus, only the powder is affected by the magnetic force.

This proposed motion-control system provides new insights into the design of new actuators and novel devices.

\section{MATERIALS AND METHODS}

\section{$\mathrm{X}$-ray tomography}

The three-dimensional morphological structure of pine cone scales was determined using X-ray micro computed tomography at the $6 \mathrm{C}$ beamline of the Pohang Accelerator Laboratory (Pohang, Korea). A $23 \mathrm{~mm}$ filter and a $1.5 \mathrm{~mm}$-thick silicon wafer were positioned along the pathway of X-ray beam propagation to minimize the photothermal damage to the test samples. The field of view was $4 \mathrm{~mm} \times 3.5 \mathrm{~mm}$, and the distance between the test sample and the camera was $20 \mathrm{~cm}$. X-ray images were acquired using a sCMOS camera with a resolution of $2560 \times 2160$ pixels (Andor Zyla, Belfast, Northern Ireland, UK). The spatial resolution, which was evaluated based on the pixel size of the camera equipped with a $\times 4$ objective lens, was $\sim 1.6 \mu \mathrm{m}$ per pixel. The test sample was fixed to a sample holder attached to a rotating stage, which was rotated from $0^{\circ}$ to $180^{\circ}$ at $0.5^{\circ}$ intervals.

Erroneous spots that appeared in the captured X-ray images were removed. A tomogram of each image was converted into a sinogram using the modified
Bronnikov algorithm filter in Octopus software (inCT, Gent, Flemish, Belgium) to achieve a reconstruction with enhanced spatial resolution.

\section{PNIPAAm synthesis}

Photocrosslinkable PNIPAAm was synthesized using the free-radical polymerization method. $\mathrm{N}$-isopropylacrylamide monomer $(100 \mathrm{mg}$; Sigma-Aldrich, St Louise, MO, USA) was dissolved in deionized water $(0.6 \mathrm{ml})$ to prepare a pre-gel solution. 2-Hydroxy-1-[4-(hydroxyethoxy)phenyl]-2-methyl-1-propanone (1 mg; Sigma-Aldrich) was added to the monomer solution as a photoinitiator. $N, N^{\prime}$-Methylenebisacrylamide (1 mg; Sigma-Aldrich) was dissolved in deionized water $(0.1 \mathrm{ml})$.

The pre-gel solution $(700 \mu \mathrm{l})$ was poured into a polydimethylsiloxane mold (Dow Corning Corp., Midland, MI, USA). Supporting beams (1 mm height) were placed along the wall of the polydimethylsiloxane mold for height equalization. The solution was covered with a thin film and irradiated with ultraviolet light at $365 \mathrm{~nm}$ (VIRVER Lourmat-4.L, Marne-la-Vallée, France) for $8 \mathrm{~min}$. Free radicals in the illuminated regions lead to polymerization.

\section{Fabrication of symmetric PNIPAAm layers containing $\mathrm{Fe}_{3} \mathrm{O}_{4}$}

The pre-gel solution $(700 \mu \mathrm{l})$ was poured over the fabricated single layer of PNIPAAm. $\mathrm{Fe}_{3} \mathrm{O}_{4}$ powder (0.02 g) (LabCloud, Gwangju, Korea) was mixed into the pre-gel solution to prepare the layers. The $\mathrm{Fe}_{3} \mathrm{O}_{4}$ powder in the pre-gel solution was aligned vertically, horizontally or diagonally using a permanent magnet ( $0.2 \mathrm{~kg}$; Needz, Seoul, Korea). Supporting beams (1 mm height) were installed along the wall of the polydimethylsiloxane mold. Ultraviolet light $(365 \mathrm{~nm})$ was irradiated over the film-covered pre-gel solution for $8 \mathrm{~min}$. After hardening, another $700 \mu$ l of pre-gel solution was poured on the hardened double layer and the process was repeated. The fabricated symmetric PNIPAAm layers with embedded $\mathrm{Fe}_{3} \mathrm{O}_{4}$ were carefully separated from the polydimethylsiloxane mold and cut into $15 \mathrm{~mm} \times 7.5 \mathrm{~mm}$ rectangular pieces. 


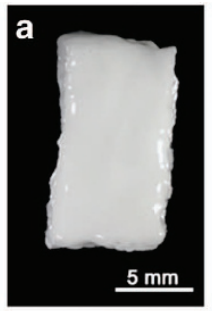

b
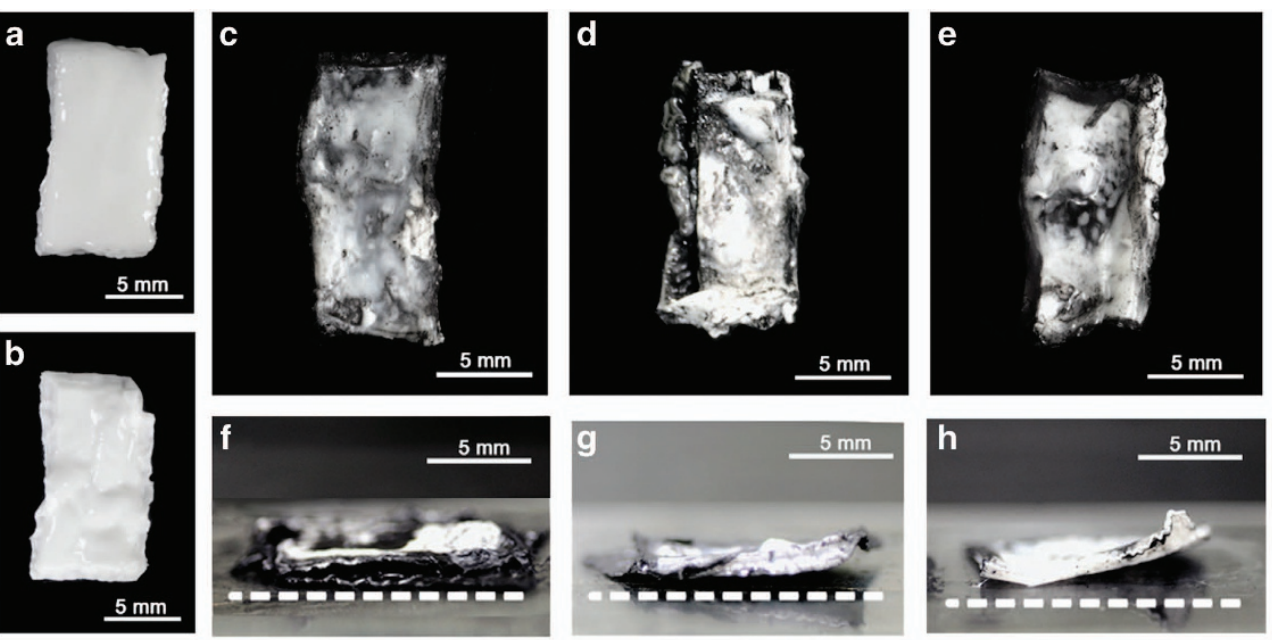

j
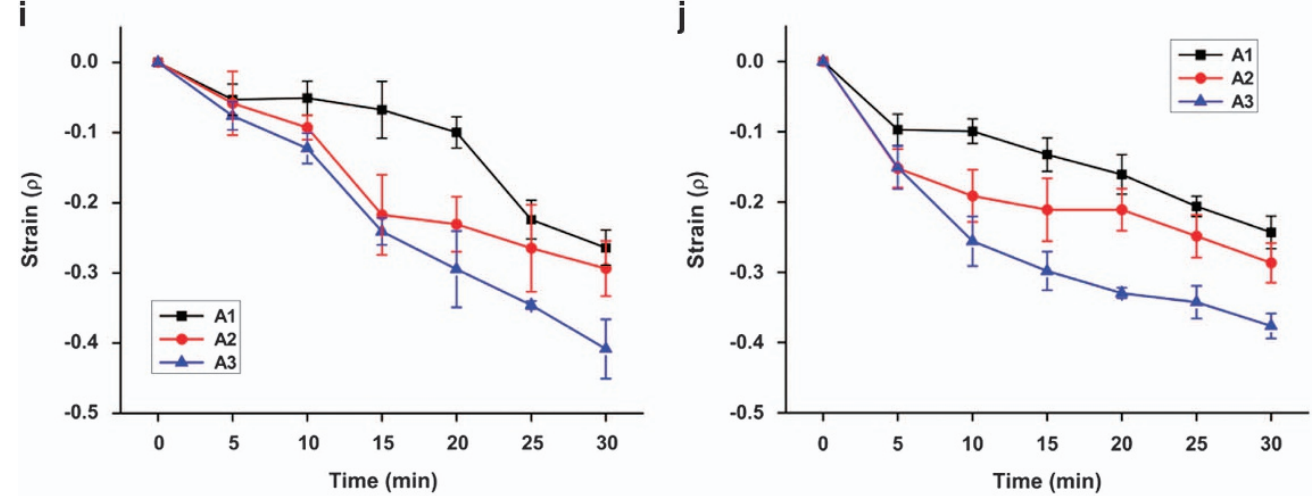

k

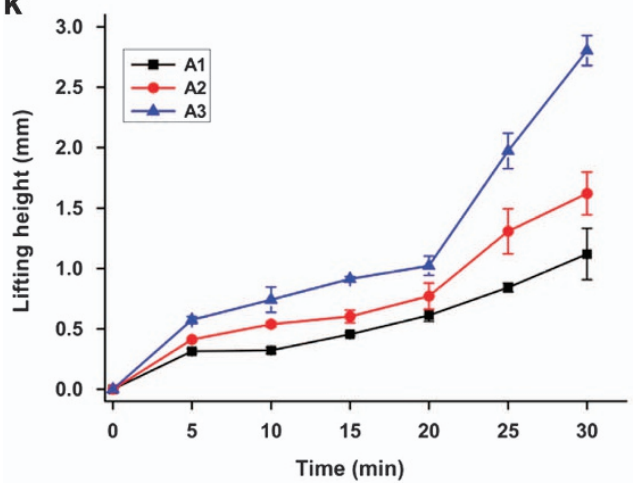

1

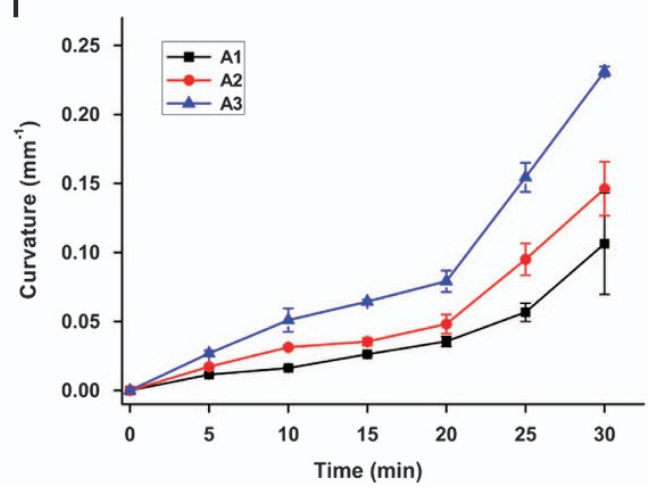

Figure 2 The bending motion of the PNIPAAm layer depends on the $\mathrm{Fe}_{3} \mathrm{O}_{4}$ content. (a) Shape of the PNIPAAm layer before heating. (b) Morphological changes in the PNIPAAm structure after heating. The PNIPAAm structure shrinks in every direction, and no bending motion was observed. (c-e) Top view of the morphological changes in PNIPAAm embedded with 0.01 (A1), 0.02 (A2) and $0.03 \mathrm{~g}$ (A3) of $\mathrm{Fe}_{3} \mathrm{O}_{4}$ after heating. Morphological changes can be clearly observed in A3. ( $\mathbf{f}-\mathbf{h})$ Side view of the morphological changes in A1, A2 and A3. A3 is considerably lifted compared with A1 and A2. (i, j) Changes in strain in A1, A2 and A3 perpendicular and parallel to the aligned $\mathrm{Fe}_{3} \mathrm{O}_{4}$. $\mathrm{A} 3$ exhibits the largest change. (k) Variation in lifting height measured from a time-lapse image. The lifting height of $\mathrm{Fe}_{3} \mathrm{O}_{4}$ in $\mathrm{A} 3$ is approximately six times greater than that of $\mathrm{A} 1$. (g) The variation in the bending curvature of $\mathrm{A} 1, \mathrm{~A} 2$ and $\mathrm{A} 3$ was calculated every 5 min. Given that A3 shows the largest lifting height, A3 shows the largest change in bending curvature. Error bars indicate the s.d. $(n=5)$. PNIPAAm, poly $(N-$ isopropylacrylamide).

\section{Heating and data acquisition}

The fabricated PNIPAAm layers were dipped into deionized water for full swelling. Given that the PNIPAAm layer was transformed at the critical temperature of $32{ }^{\circ} \mathrm{C}$, the fully hydrated PNIPAAm layers were heated to $40{ }^{\circ} \mathrm{C}$ using a hot plate. The transformation of the PNIPAAm layer was photographed using a digital camera (Nikon D700, Tokyo, Japan) at $10 \mathrm{~s}$ intervals for $30 \mathrm{~min}$. The acquired data were quantitatively analyzed using Image J software (National Institutes of Health, Bethesda, MD, USA) to calculate the changes in strain and surface area.
Scanning electron microscope experiment

The detailed morphological structures of $\mathrm{Fe}_{3} \mathrm{O}_{4}$ powder and PNIPAAm with $\mathrm{Fe}_{3} \mathrm{O}_{4}$ were observed using a field-emission scanning electron microscope (JEOL JSM-7401F, JEOL, Tokyo, Japan). For observation, PNIPAAm with $\mathrm{Fe}_{3} \mathrm{O}_{4}$ was freeze-dried at $-84^{\circ} \mathrm{C}$ using a freeze drying system (LABCONCO, Kansas City, MO, USA) for $24 \mathrm{~h}$. The freeze-dried samples were attached to the sample holder and observed at various magnifications. 


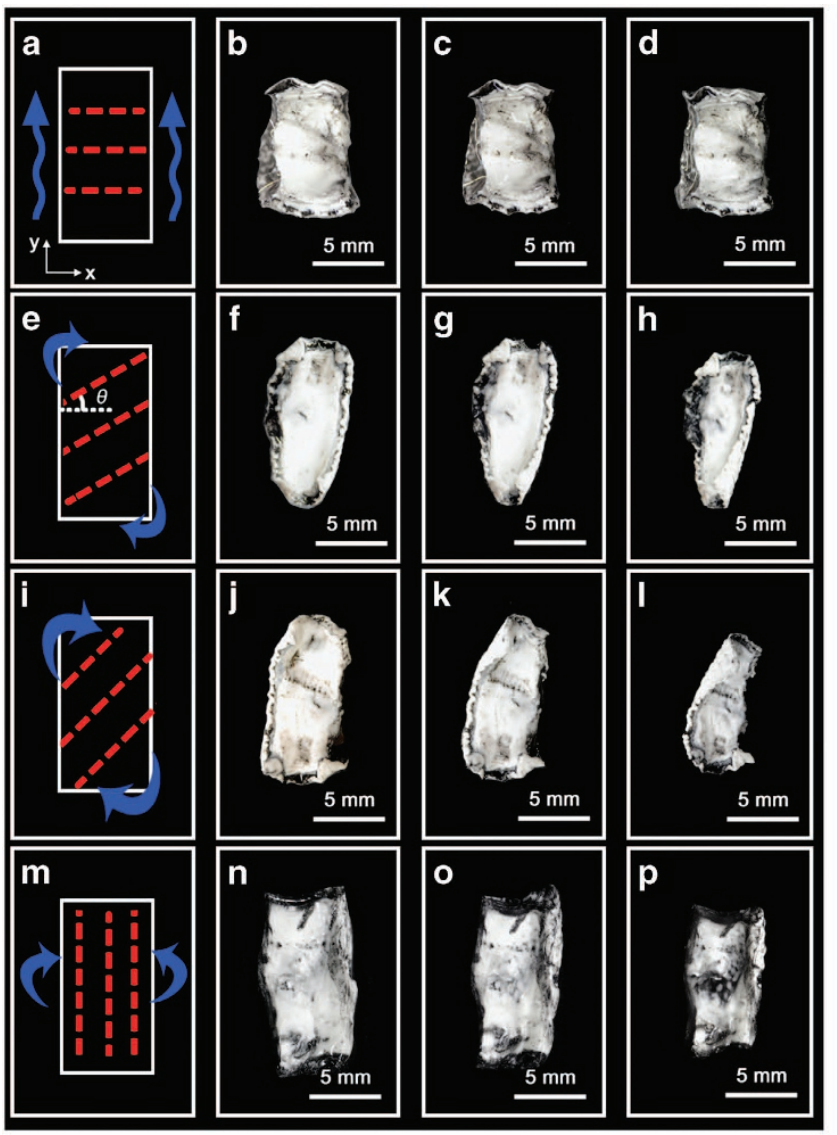

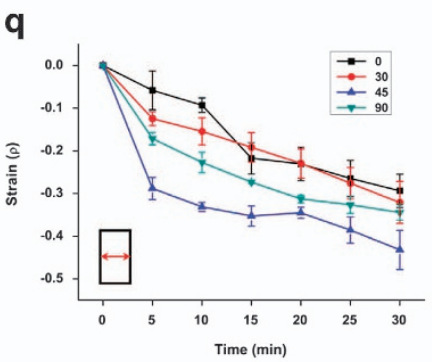

$\mathbf{S}$
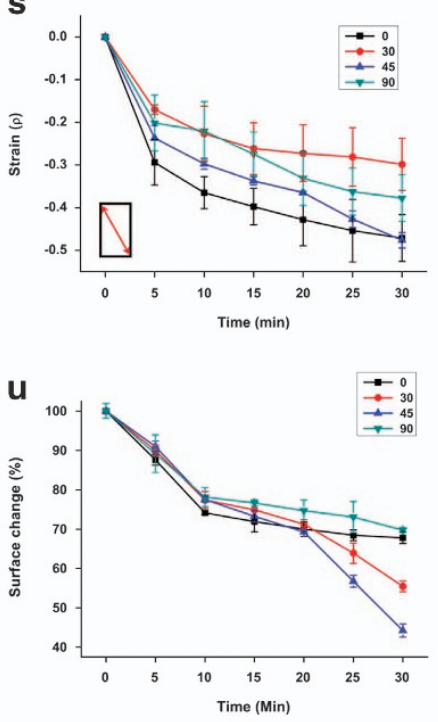

t

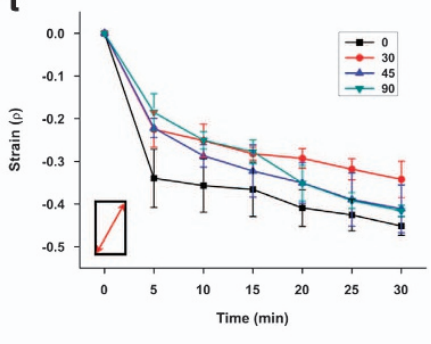

$r$
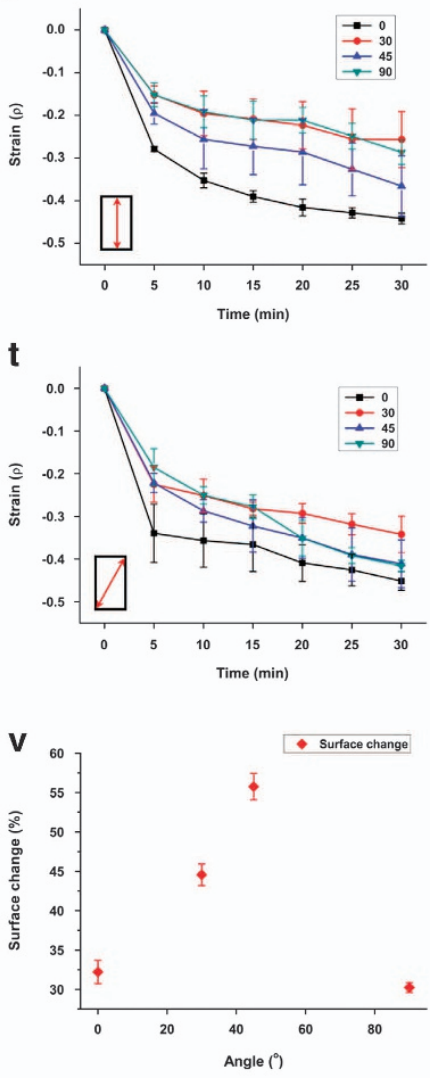

Figure 3 Bending direction according to the alignment angle $\theta$ of $\mathrm{Fe}_{3} \mathrm{O}_{4}$. (a-d) Morphological changes in PNIPAAm layers at $\theta=0^{\circ}$. The PNIPAAm layers shrink vertically in an accordion-like manner. (e-h) Morphological changes in PNIPAAm layers at $\theta=30^{\circ}$. The edges roll up in the diagonal direction. (i-I) Morphological changes in PNIPAAm layers at $\theta=45^{\circ}$. The hydrogel layers are twisted in the diagonal direction. ( $\left.\mathbf{m}-\mathbf{p}\right)$ Morphological changes in PNIPAAm layers at $\theta=90^{\circ}$. As shown in Figure 2, the layers are lifted. (q-t) Variations in strain measured every $5 \mathrm{~min}$ in the parallel, perpendicular and diagonal directions. Changes in strain in the layers differ from each other greatly because the PNIPAAm layers bend in different directions. (u, v) Temporal variations in the surface area of the layers. The layers with aligned $\mathrm{Fe}_{3} \mathrm{O}_{4}$ at $\theta=45^{\circ}$ exhibit the largest change, and thus, their corresponding surface areas are greatly changed. The layers with $\theta=0^{\circ}$ and $90^{\circ}$ show smaller changes in surface area than the other layers. Error bars indicate the s.d. $(n=5)$. PNIPAAm, poly $(N$-isopropylacrylamide).

\section{Magnetic hysteresis curve measurement}

The magnetic field-dependent d.c. magnetization of pure $\mathrm{Fe}_{3} \mathrm{O}_{4}$ powder and fully dried PNIPAAm with $\mathrm{Fe}_{3} \mathrm{O}_{4}$ was measured using the vibrating sample magnetometer of the Quantum Design Physical Property Measurement System (PPMS DynaCool 9T, San Diego, CA, USA) at room temperature. Pure $\mathrm{Fe}_{3} \mathrm{O}_{4}$ powder was packed in a vibrating sample magnetometer capsule for measurement, and the diamagnetic background of the capsule $\left(-1.4 \times 10^{-8}\right.$ e.m. u. $\mathrm{Oe}^{-1}$ ) was subtracted from the measured moment. The dried PNIPAAm with $\mathrm{Fe}_{3} \mathrm{O}_{4}$ powder was attached to the sample holder for measurement. For comparing the moment per unit mass, the measured moment data were divided by the mass.

\section{Evaluation of Young's modulus}

The variation in Young's modulus as a function of the amount and alignment angle of $\mathrm{Fe}_{3} \mathrm{O}_{4}$ in the PNIPAAm layer was evaluated using a universal testing machine (Instron 3344, Norwood, MA, USA). The dimensions of the test samples were measured before and after performing a tensile experiment to calculate the Young's modulus of the samples. The lower portion of the sample was fixed with clamps and pulled upward at a speed of $5 \mathrm{~mm} \mathrm{~min}^{-1}$. The Young's modulus was calculated using the following formula:

$$
E=\frac{\sigma}{\varepsilon}=\frac{F / A}{\Delta L / L}
$$

where $E, \sigma$ and $\varepsilon$ denote the Young's modulus of the test sample and the stress and strain exerted on the sample, respectively. $F$ is the force measured by a load cell and $A$ is the tension acting on the surface area. $L$ denotes the initial length of the sample and $\Delta L$ is the extended length.

\section{RESULTS}

Lifting height based on $\mathrm{Fe}_{3} \mathrm{O}_{4}$ content

A bending gradient is controlled mainly by controlling the amount of $\mathrm{Fe}_{3} \mathrm{O}_{4}$. Pure PNIPAAm shrinks in every direction in response to heat (Figures $2 \mathrm{a}$ and $\mathrm{b}$ ). However, the shrinking direction of the PNIPAAm layer with aligned $\mathrm{Fe}_{3} \mathrm{O}_{4}$ follows a bending motion: as different expansion directions of tissues and fibrils in pine cone scales lead to motion, the aligned $\mathrm{Fe}_{3} \mathrm{O}_{4}$ disturbs the shrinkage in the parallel direction and facilitates motion of the hydrogel. The bending gradient varies depending on the amount of $\mathrm{Fe}_{3} \mathrm{O}_{4}(0.01$ (A1), 0.02 (A2) and $0.03 \mathrm{~g}$ (A3)) embedded into the PNIPAAm layer. The higher the amount of $\mathrm{Fe}_{3} \mathrm{O}_{4}$ the larger is the bending motion. $\mathrm{A} 3$ shows the highest lifting motion and thus displays the largest change in strain (Figures $2 \mathrm{i}$ and $\mathrm{j}$ ). As the $\mathrm{Fe}_{3} \mathrm{O}_{4}$ content is increased by 2- or 3 -fold, the lifting height increases 1.5 - or 2.5-fold, respectively. Similar to the results for strain factors, A3 exhibits the biggest bending curve. When the amount of $\mathrm{Fe}_{3} \mathrm{O}_{4}$ is increased 2- or 3-fold, the corresponding curves increase 1.4- or 2.1-fold, respectively.

These differences in bending motion are apparently induced by different tensile strengths caused by varying amounts of $\mathrm{Fe}_{3} \mathrm{O}_{4}$. 
Young's modulus of the pure PNIPAAm layer without $\mathrm{Fe}_{3} \mathrm{O}_{4}$ is $57.2 \pm 4.6 \mathrm{kPa}$. The PNIPAAm layer containing $\mathrm{Fe}_{3} \mathrm{O}_{4}$ displays a higher Young's modulus. The Young's modulus of the Al and the
A3 layers is $62.0 \pm 4.9$ and $64.7 \pm 4.5 \mathrm{kPa}$, respectively. Before heating, the Young's modulus of $\mathrm{A} 3$ is slightly higher than that of A1. After heating, because the $\mathrm{Fe}_{3} \mathrm{O}_{4}$ powder maintains the shape, the Young's
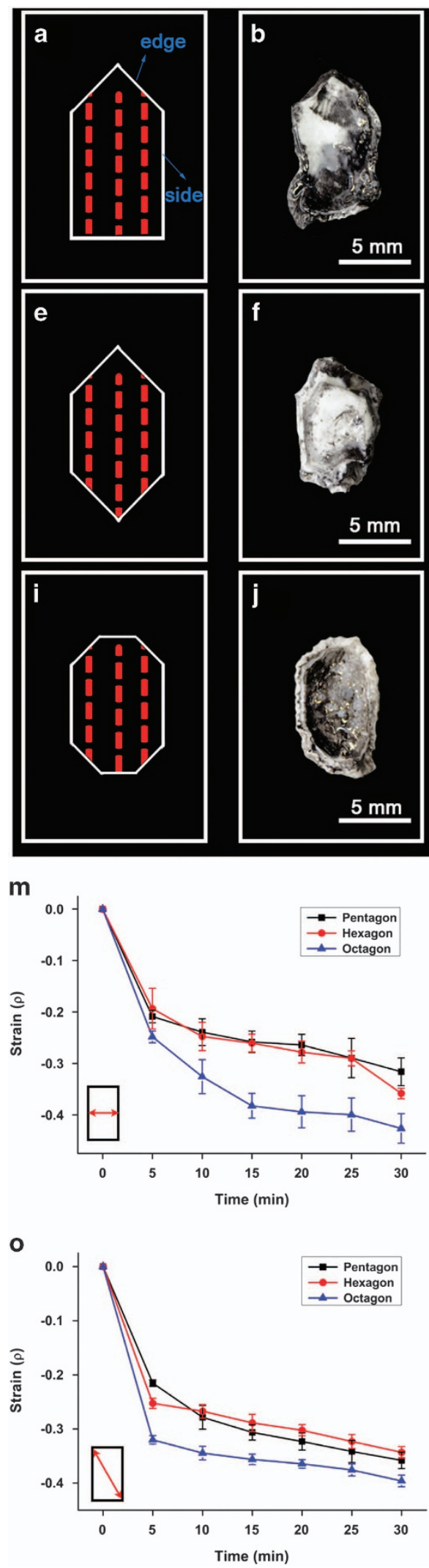
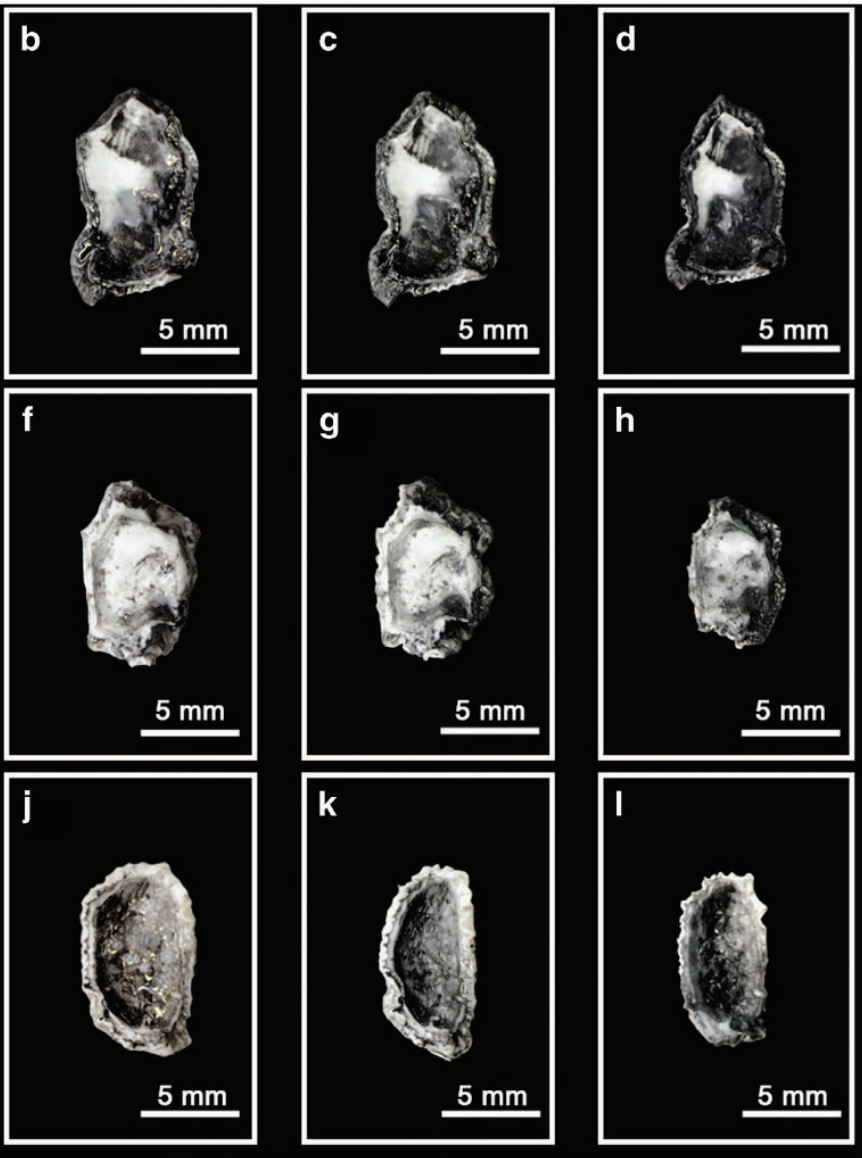

n

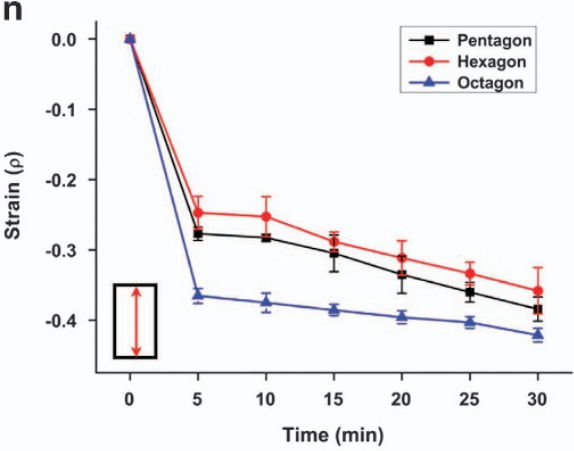

p

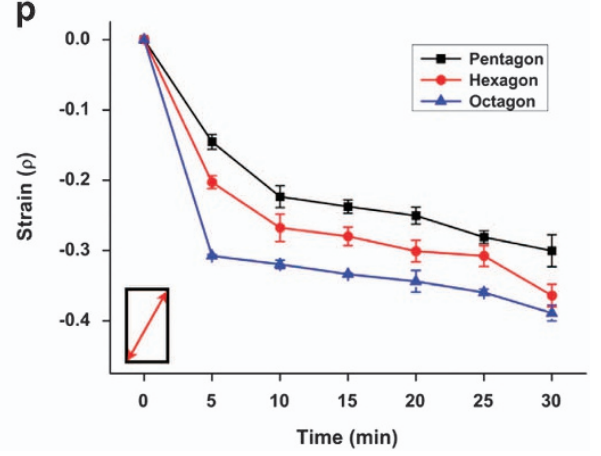


Figure 4 Morphological deformation based on the shape of the layer. (a-d) When the layer is cut into a pentagonal structure, the diagonal edges roll up and meet, and the parallel side rolls up and is lifted upward. (e-h) When the layer is cut into a hexagonal structure, the diagonal edges roll up and meet. (i-I) When the layer is cut into an octagonal structure, the diagonal edges roll up and meet, similar to the pentagon and hexagon cases. Given that the number of edges in an octagon is higher than in a hexagon, a bowl-shaped structure is formed when the edges of the former meet. The shapes of the octagonal structure before/after heating are similar to those of a pine cone scale before/after bending, respectively. ( $\mathbf{m}-\mathbf{p})$ Variations in strain measured every $5 \mathrm{~min}$ in the parallel, perpendicular and diagonal directions. Given that increasing the number of sides increases the structural deformation, the octagon demonstrates the largest changes in strain in every direction. The error bars indicate the s.d. $(n=5)$.

modulus of A3 rapidly increases $(141.6 \pm 12.9 \mathrm{kPa})$. Similar to A3, the Young's modulus of $\mathrm{A} 1$ also increases, but it is less than that of A3 $(112.8 \pm 11.7 \mathrm{kPa})$. Because the Young's modulus of A3 is larger than that of A1, A3 exerts a larger impact than A1 and A3 exhibits a greater transformation than $\mathrm{A} 1$. The lifting height and bending curve can be easily controlled by adjusting the $\mathrm{Fe}_{3} \mathrm{O}_{4}$ content.

\section{Bending direction based on alignment angle}

The folding direction of the PNIPAAm layer can be changed simply by varying the alignment direction of $0.02 \mathrm{~g}$ of $\mathrm{Fe}_{3} \mathrm{O}_{4}$ (A2). We changed the alignment angle $\theta$ to $0^{\circ}, 30^{\circ}, 45^{\circ}$ and $90^{\circ}$, and observed the corresponding bending motions. When $\theta=0^{\circ}$, the PNIPAAm shrinks vertically, similar to an accordion (Figures $3 \mathrm{a}-\mathrm{d}$ ). A minimum contraction along the $x$ direction was observed because addition of $\mathrm{Fe}_{3} \mathrm{O}_{4}$ disrupts the shrinkage of the layer in this direction (Figure $3 q$ ). PNIPAAm primarily shrinks in the $y$ direction rather than the $x$ direction (Figure $3 \mathrm{r}$ ). Moreover, $\mathrm{Fe}_{3} \mathrm{O}_{4}$ addition changes the diagonal strain greatly. When $\theta=30^{\circ}$, the corner of the PNIPAAm forms a large fold and the side edge rolls diagonally (Figures $3 \mathrm{e}-\mathrm{h}$ ). Similar to the case of $\theta=30^{\circ}$, the overall motion for $\theta=45^{\circ}$ is largely circular and twisted (Figures $3 \mathrm{i}-1$ ). The bending motion is largest at $\theta=45^{\circ}$, and thus, the surface area of the PNIPAAm layer exhibits the largest change (Figures $3 \mathrm{u}$ and v). When $\theta=90^{\circ}$, the layer shows a lifting motion (Figures $3 \mathrm{~m}-\mathrm{p}$ ).

Interestingly, the direction of bending motion is mainly affected by the alignment angle of $\mathrm{Fe}_{3} \mathrm{O}_{4}$. The bending direction is perpendicular to the alignment angle of the $\mathrm{Fe}_{3} \mathrm{O}_{4}$ because the aligned $\mathrm{Fe}_{3} \mathrm{O}_{4}$ serves as a frame. It is also closely related to tensile strength. The tensile strength in the direction parallel to the aligned $\mathrm{Fe}_{3} \mathrm{O}_{4}$ powder is $64.7 \pm 4.5 \mathrm{kPa}$, and that in the direction perpendicular to the aligned $\mathrm{Fe}_{3} \mathrm{O}_{4}$ is $25.4 \pm 4.7 \mathrm{kPa}$; the Young's modulus in the perpendicular direction is much smaller than that in the parallel direction. This result implies that the layer can be easily transformed (shrunk in this experiment) in the perpendicular direction. This phenomenon explains why the layer bends in different directions depending on the alignment angle: alignment angles of $0^{\circ}, 30^{\circ}, 45^{\circ}$ and $90^{\circ}$ are $90^{\circ}, 120^{\circ}, 135^{\circ}$ and $180^{\circ}$, respectively. In addition, the lower layers (pure PNIPAAm) shrink in every direction, but the layer containing $\mathrm{Fe}_{3} \mathrm{O}_{4}$ powder does not shrink in the direction parallel to the aligned $\mathrm{Fe}_{3} \mathrm{O}_{4}$ powder; thus, the layer is lifted in the direction parallel to the aligned $\mathrm{Fe}_{3} \mathrm{O}_{4}$.

Thus, when $\theta=0^{\circ}$, the shrinkage in the $y$ direction (the direction perpendicular to the aligned $\mathrm{Fe}_{3} \mathrm{O}_{4}$ ) is greater than that in $x$ direction: the strain change in the $y$ direction is $\sim 1.5$ times larger than that in the $x$ direction (Figures $3 \mathrm{q}$ and $\mathrm{r}$ ). When $\theta=90^{\circ}$, the shrinkage in the $x$ direction (the direction perpendicular to the aligned $\mathrm{Fe}_{3} \mathrm{O}_{4}$ ) is slightly greater than that in the $y$ direction, and the strain change in the $x$ direction is greater than that in the $y$ direction. The perpendicular direction of $\theta=0^{\circ}$ is the $y$ direction that has twice the length. This signifies that the layers have space for shrinkage in the $y$ direction. Because the length change measurements were performed with a top-down view, the bending also affects the length change: when bending occurs, the length in the $y$ direction shortens. Thus, the strain change difference between the $x$ and $y$ directions of a $90^{\circ}$ angle (1.2 times) is smaller than that of a $0^{\circ}$ angle (1.5 times). Because $\theta=45^{\circ}$ results in largely circular PNIPAAm that is twisted along the diagonal axis, the $45^{\circ}$ angle strain changes are greater in every direction than those of the $30^{\circ}$ angle (Figures $3 \mathrm{q}-\mathrm{t}$ ).

\section{Bending shape based on the shape of the structure}

When the structure is not rectangular, the shape of the layer is slightly deformed even if the alignment angle and the composition of $\mathrm{Fe}_{3} \mathrm{O}_{4}$ are the same $\left(\theta=0^{\circ}, 0.02 \mathrm{~g} \mathrm{Fe}_{3} \mathrm{O}_{4}(\mathrm{~A} 2)\right)$.

When there is a layer edge of a certain length, the edge folds slightly and forms a low wall. In a pentagonal structure, the upper sides roll along the edge and the lower side is lifted as in the rectangular structure (Figures $4 \mathrm{a}-\mathrm{d}$ ). The middle portion has a concave configuration because the edge of the hexagon layer is also rolled up (Figures $4 \mathrm{e}-\mathrm{h}$ ). An octagonal structure is similar to a pine cone scale, and its eight edges roll up and meet (Figures 4i-1).

The center of the layers primarily shrinks and the largest surface area differences are between the bottom and middle layers, causing a concave shape. The edges come together without any spaces like a connected wall structure. This phenomenon is attributed to shrinkage starting from the periphery toward the center of the layer that causes the edges to meet and form a bowl-shaped structure.

The transformed octagonal structure is similar to the concave structure of the scale of a closed pine cone. This octagonal structure is greatly deformed, and the largest changes in strain in every direction were observed in this structure (Figures $4 \mathrm{~m}-\mathrm{p}$ ). An increase in the side length leads to higher strain changes. This signifies that the number of sides affects the transformation rate.

\section{DISCUSSION}

Bilayer structures containing two different chemical components have been widely used for motion control ${ }^{4,6,7,14,25-29}$. Recently, a simple method for inducing bending motion of a symmetric structure was introduced. However, it was difficult to control the direction of motion ${ }^{35}$. Differing from previous research, $\mathrm{Fe}_{3} \mathrm{O}_{4}$ is inserted into homogenous layers of the pine cone scale-inspired proposed structure to induce various motions: a simple method was adopted to control the motion of symmetric structures. We used PNIPAAm as the stimuli-responsive hydrogel; however, any chemical component can be used to induce dynamic motion.

The amount and alignment angle of $\mathrm{Fe}_{3} \mathrm{O}_{4}$ and the shape of the hydrogel layers are key elements in controlling the bending gradient, folding direction and transformation, respectively. Given that the aligned $\mathrm{Fe}_{3} \mathrm{O}_{4}$ powder acts as a bone structure of the PNIPAAm layer, it prohibits folding motion in the direction perpendicular to the aligned $\mathrm{Fe}_{3} \mathrm{O}_{4}$ and encourages lifting motion in the direction parallel to the aligned $\mathrm{Fe}_{3} \mathrm{O}_{4}$. The amount of embedded $\mathrm{Fe}_{3} \mathrm{O}_{4}$ is proportional to the strength of prevention. Thus, increasing the $\mathrm{Fe}_{3} \mathrm{O}_{4}$ can prevent more bending motion in the direction perpendicular to the aligned $\mathrm{Fe}_{3} \mathrm{O}_{4}$. In addition, the folding direction of the layer can be easily changed by varying the $\mathrm{Fe}_{3} \mathrm{O}_{4}$ alignment angle. The final configuration of the 
structure can be determined by changing the shape of the hydrogel structure.

This transformation is strongly associated with the shape of the structure and the $\mathrm{Fe}_{3} \mathrm{O}_{4}$ alignment angle. The octagonal shape and the parallel alignment of fibers lead to the formation of a concave structure and to the folding motion of the pine cone scales. The curvature of the petals also has an important role in folding and blooming of flowers ${ }^{36,37}$. In addition, octagonal or rounded petals have lines that are similar to the fibers in pine cone scales and may influence flower blooming.

The curvature of the deformed pine cone scales allows the scales to fit with adjacent scales on its outer, bottom, left and right sides. Without the curvature, the folding scales become parallel to each other with a gap at the tip of a pine cone. This finding demonstrates that pine cone scales exhibit an appropriate morphological structure to create a gap-free covering for pine seeds that prevents water penetration.

The folding motion of pine cone scales is an interesting scientific discovery, and the bio-inspired hydrogel layers used in this study can be applied in diverse fields that require motion control. In addition, various types of dynamic motion can be easily controlled at a low cost.

\section{CONFLICT OF INTEREST}

The authors declare no conflict of interest.

\section{ACKNOWLEDGEMENTS}

We are grateful to the Pohang Accelerator Laboratory (Pohang, Korea) and the Molecular Biotechnology Laboratory of Pohang University of Science and Technology for their assistance in X-ray imaging experiments performed at the 6C beamline and for tensile strength measurement, respectively. We also appreciate the National Institute for Nanomaterials Technology (Pohang, Korea) and Dr Tae-Hwan Jang and Prof Jae-Hoon Park at the Center for complex phase materials of Max Plank POSTECH who performed the scanning electron microscopy experiments and magnetic field-dependent d.c. magnetization of samples. This work was supported by a National Research Foundation of Korea (NRF) grant funded by the Korean government (MSIP) (No. 2016R1E1A2A01939202).

Author contributions: KS and SJL proposed the study. KS performed the experiment, analyzed the data and wrote the paper. Both authors discussed the results and contributed to the preparation of the manuscript.

1 Roy, D., Cambre, J. N. \& Sumerlin, B. S. Future perspectives and recent advances in stimuli-responsive materials. Prog. Polym. Sci. 35, 278-301 (2010).

2 Ehrick, J. D., Deo, S. K., Browning, T. W., Bachas, L. G., Madou, M. J. \& Daunert, S. Genetically engineered protein in hydrogels tailors stimuli-responsive characteristics. Nat. Mater. 4, 298-302 (2005)

3 Lendlein, A., Jiang, H., Jünger, O. \& Langer, R. Light-induced shape-memory polymers. Nature 434, 879-882 (2005).

4 Geryak, R. \& Tsukruk, V. V. Reconfigurable and actuating structures from soft materials. Soft Matter 10, 1246-1263 (2014).

5 Miyata, T., Asami, N. \& Uragami, T. A reversibly antigen-responsive hydrogel. Nature 399, 766-769 (1999).

6 Tokarev, I. \& Minko, S. Stimuli-responsive hydrogel thin films. Soft Matter $\mathbf{5}$ 511-524 (2009).

7 Prabaharan, M. \& Mano, J. F. Stimuli-responsive hydrogels based on polysaccharides incorporated with thermo-responsive polymers as novel biomaterials. Macromol. Biosci. 6, 991-1008 (2006)

8 Stuart, M. A. C., Huck, W. T., Genzer, J., Müller, M., Ober, C., Stamm, M. Sukhorukov, G. B., Szleifer, I., Tsukruk, V. V. \& Urban, M. Emerging applications of stimuli-responsive polymer materials. Nat. Mater. 9, 101-113 (2010).

9 de Las Heras Alarcon, C., Pennadam, S. \& Alexander, C. Stimuli responsive polymers for biomedical applications. Chem. Soc. Rev. 34, 276-285 (2005).
10 Mano, J. F. Stimuli-responsive polymeric systems for biomedical applications. Adv. Eng. Mater. 10, 515-527 (2008).

$11 \mathrm{Kim}, \mathrm{H}$. \& Lee, S. J. Stomata-inspired membrane produced through photopolymerization patterning. Adv. Funct. Mater. 25, 4496-4505 (2015).

12 Qiu, Y. \& Park, K. Environment-sensitive hydrogels for drug delivery. Adv. Drug Deliv. Rev. 64, 49-60 (2012).

13 Liu, D., Bastiaansen, C. W., den Toonder, J. M. \& Broer, D. J. Single-composition threedimensionally morphing hydrogels. Soft Matter 9, 588-596 (2013).

14 Lee, H., Xia, C. \& Fang, N. X. First jump of microgel; actuation speed enhancement by elastic instability. Soft Matter 6, 4342-4345 (2010).

15 Jeong, B. \& Gutowska, A. Lessons from nature: stimuli-responsive polymers and their biomedical applications. Trends Biotechnol. 20, 305-311 (2002).

16 O'Grady, M. L., Kuo, P.-L. \& Parker, K. K. Optimization of electroactive hydrogel actuators. ACS Appl. Mater. Interfaces 2, 343-346 (2009).

17 Haraguchi, K., Murata, K. \& Takehisa, T. Stimuli-responsive nanocomposite gels and soft nanocomposites consisting of inorganic clays and copolymers with different chemical affinities. Macromolecules 45, 385-391 (2011).

$18 \mathrm{Hu}, \mathrm{Z}$., Zhang, X. \& Li, Y. Synthesis and application of modulated polymer gels. Science 269, 525 (1995).

19 Ionov, L. Hydrogel-based actuators: possibilities and limitations. Mater. Today 17 494-503 (2014).

20 Wong, W. S., Li, M., Nisbet, D. R., Craig, V. S., Wang, Z. \& Tricoli, A. Mimosa Origami: a nanostructure-enabled directional self-organization regime of materials. Sci. Adv. 2, e1600417 (2016)

21 Harmon, M. E., Tang, M. \& Frank, C. W. A microfluidic actuator based on thermoresponsive hydrogels. Polymer 44, 4547-4556 (2003).

22 Kumar, K., Knie, C., Bléger, D., Peletier, M. A., Friedrich, H., Hecht, S., Broer, D. J. Debije, M. G. \& Schenning, A. P. A chaotic self-oscillating sunlight-driven polymer actuator. Nat. Commun. 7, 11975 (2016).

23 Thérien-Aubin, H., Wu, Z. L., Nie, Z. \& Kumacheva, E. Multiple shape transformations of composite hydrogel sheets. J. Am. Chem. Soc. 135, 4834-4839 (2013).

24 Shim, T. S., Kim, S. H., Heo, C. J., Jeon, H. C. \& Yang, S. M. Controlled origami folding of hydrogel bilayers with sustained reversibility for robust microcarriers. Angew. Chem. Int. Ed. 51, 1420-1423 (2012).

25 Liu, L., Jiang, S., Sun, Y. \& Agarwal, S. Giving direction to motion and surface with ultra-fast speed using oriented hydrogel fibers. Adv. Funct. Mater. 26, 1021-1027 (2016).

26 Kelby, T. S., Wang, M. \& Huck, W. T. Controlled folding of 2D Au-polymer brush composites into 3D microstructures. Adv. Funct. Mater. 21, 652-657 (2011).

$27 \mathrm{Ma}$, Y. \& Sun, J. Q. Humido- and thermo-responsive free-standing films mimicking the petals of the morning glory flower. Chem. Mater. 21, 898-902 (2009).

28 Bassik, N., Abebe, B. T., Laflin, K. E. \& Gracias, D. H. Photolithographically patterned smart hydrogel based bilayer actuators. Polymer 51, 6093-6098 (2010).

29 Stoychev, G., Puretskiy, N. \& Ionov, L. Self-folding all-polymer thermoresponsive microcapsules. Soft Matter 7, 3277-3279 (2011).

30 Dawson, C., Vincent, J. F. V. \& Rocca, A. M. How pine cones open. Nature 390 668-668 (1997).

31 Bar-On, B., Sui, X., Livanov, K., Achrai, B., Kalfon-Cohen, E., Wiesel, E. \& Wagner, H. D. Structural origins of morphing in plant tissues. Appl. Phys. Lett. 105, 033703 (2014).

32 Harlow, W. M., Cote, W. \& Day, A. The opening mechanism of pine cone scales. J. For. 62, 538-540 (1964).

33 Song, K., Yeom, E., Seo, S.-J., Kim, K., Kim, H., Lim, J.-H. \& Lee, S. J. Journey of water in pine cones. Sci. Rep. 5, 9963 (2015).

34 Reyssat, E. \& Mahadevan, L. Hygromorphs: from pine cones to biomimetic bilayers J. R. Soc. Interface 6, 951-957 (2009).

35 Song, K., Chang, S.-S., Roper, M., Kim, H. \& Lee, S. J. A biologically-inspired symmetric bidirectional switch. PLOS ONE 12, e0169856 (2017).

36 Liang, H. \& Mahadevan, L. Growth, geometry, and mechanics of a blooming lily. Proc. Natl Acad. Sci. USA 108, 5516-5521 (2011).

37 Amar, M. B., Müller, M. M. \& Trejo, M. Petal shapes of sympetalous flowers: the interplay between growth, geometry and elasticity. New J. Phys. 14, 085014 (2012). Attribution 4.0 International License. The images or party material in this article are included in the article's other third party material in this article are included in the article's Creative Commons license, unless indicated otherwise in the credit line; if the material is not included under the Creative Commons license, users will need to obtain permission from the license holder to reproduce the material. To view a copy of this license, visit http:// creativecommons.org/licenses/by/4.0/

(C) The Author(s) 2017 